\title{
Desarrollo y Validación de una Escala de Miedos del Mentor en Contexto Universitario
}

\author{
Development and Validation of a Mentor Fears' Scale in University Context
}

\author{
Miguel Aurelio Alonso García ${ }^{1}$ y Silvia Andrea Sánchez-Herrero Arbide ${ }^{2}$
}

\begin{abstract}
Resumen
El objetivo es crear y validar una escala que permita evaluar los miedos de los mentores en entornos universitarios. Se parte de una escala inicial de 25 elementos que se aplica a una muestra de 748 estudiantes universitarios. Tras realizar un análisis factorial exploratorio con la mitad de la muestra se obtienen cuatro factores que se confirman con un análisis factorial confirmatorio, utilizando la otra mitad. Las dimensiones encontradas son "miedo a equivocarme", "miedo a no saber qué va a pasar", "miedo a que no me respeten" y "miedo al superior". Los análisis muestran una invarianza factorial estricta en función del género y la experiencia previa como mentor, encontrándose mayores miedos en mujeres y en personas que nunca habían ejercido el rol. Cuanto mayor es el miedo, menor es la autoeficacia y las habilidades sociales. Se discuten las implicaciones y posibles intervenciones en los programas de mentoría en la universidad.
\end{abstract}

Palabras clave: miedo, mentor, mentoría, escala de miedos, evaluación

\begin{abstract}
The objective is to create and validate a scale that allows evaluating the fears of mentors in universities mentoring programmes. An initial scale of 25 items is applied to a sample of 748 university students who play the role of mentors. After undertaking an exploratory factorial analysis with half of the sample we obtain four factors that are confirmed with a confirmatory factorial analysis using the other half of the sample. The dimensions found are "fear of being mistaken", fear of not knowing what's going to happen", "fear of not being respected" and "fear of the superior". The analyses show a strict factorial invariance in terms of gender and previous experience as a mentor. Women and unexperienced mentors have more fear. In the same way, the larger the fear, the smaller the mentors'self-efficacy and social abilities are. Implications and possible interventions in mentoring university programmes are discussed.
\end{abstract}

Keywords: fears, mentor, mentoring programme, fear scale, evaluation

\footnotetext{
${ }^{1}$ Doctor en Psicología. Profesor Contratado Doctor. Departamento de Psicología Social, del Trabajo y Diferencial. Facultad de Psicología. Universidad Complutense de Madrid, España. Correo: malonsog@ucm.es

${ }^{2}$ Doctora en Psicología. Profesora Titular de Universidad. Departamento de Psicología Social, del Trabajo y Diferencial. Facultad de Psicología. Universidad Complutense de Madrid, España. Correo: sasanche@psi.ucm.es
} 


\section{Introducción}

El miedo en el lugar de trabajo lo definen Bedoya y García (2016, p. 69) como aquella emoción o sentimiento desagradable que surge cuando un individuo ha percibido una amenaza o un peligro que puede ser real o imaginario, asociado a fenómenos del trabajo, y reacciona ante ello de forma emocional, cognitiva, fisiológica y conductualmente.

Entre las consecuencias negativas del miedo para las organizaciones y sus trabajadores están la poca comunicación entre compañeros, la menor creatividad, el menor aprendizaje organizacional, el incremento de los índices de absentismo y rotación externa, la mayor insatisfacción laboral, el aumento en la resistencia al cambio, niveles de ansiedad altos (Bedoya \& García, 2016, Conroy, 2003, Kish-Gepharta, Detert, Treviño, \& Edmonson, 2009). Según Goldsmith (2008) el primer paso para vencer el miedo es aprender a identificar su existencia.

El miedo en el contexto laboral parece ser un constructo multidimensional. Así, Jericó (2006) distingue entre distintos tipos de miedos en entorno organizacional: el miedo a la no supervivencia (asociado a necesidades básicas y que deriva en miedo a perder el trabajo y a no llegar a fin de mes), miedo al rechazo (unido a la necesidad de afiliación y que deriva en el miedo a ser distinto, miedo al éxito o a destacar, y miedo a relacionarse con otras personas), miedo al fracaso (unido a la necesidad de logro y al miedo al error, a asumir riesgos, a tomar decisiones, a no ser reconocido por el trabajo), a la pérdida de poder (unido a la necesidad de poder y al miedo a perder un puesto de influencia, a no ser reconocido socialmente) y el miedo al cambio (relacionado con varias necesidades y con el miedo a un cambio de función o de localización).

Cuando en una experiencia significativa la persona se siente evaluada y cree que no puede tener éxito, se habla de miedo al fracaso (Conroy, 2001). El miedo al fracaso está presente especialmente en entornos en los que se exigen determinados resultados, p. ej., laboral, académico, deportivo, interpretación... En estudiantes el miedo al fracaso tiene un impacto mínimo en los resultados cuando se combina con un fuerte deseo de sobresalir en clase y dominar el contenido; pero cuando disminuye la orientación al éxito, el miedo al fracaso puede tener graves consecuencias para el rendimiento académico y se asocia con absentismo escolar, logros académicos más pobres e incluso el rechazo total a seguir estudiando (De Castella, Byrne, \& Covington, 2013).

Otro de los miedos es el miedo a la evaluación negativa, se trata de una aprensión hacia la evaluación por parte de los demás, el malestar ante las evaluaciones negativas, y expectativas que una persona puede tener acerca de que los otros la evaluarán negativamente (Watson \& Friend, 1969). Según Leary (1983) la predisposición a experimentar miedo a la evaluación negativa tiene al menos dos implicaciones, una de tipo subjetivo ("experiencia subjetiva" de ansiedad) y otra de tipo comportamental ("consecuencias conductuales"). Este miedo a la evaluación negativa se ha contrastado en deportistas cuando están en situaciones de alta presión psicológica (Molina, Chorot, Valiente, \& Sandín, 2014), en actividades desarrolladas en las clases de Educación Física (Torregrosa, Murcia, \& Moreno, 2015), y en personas que tienen que hablar en público (Gallego, Botella, García-Palacios, Quero, \& Baños, 2010; Orejudo, Fernández-Turrado, \& Briz, 2012).

Por lo tanto, el miedo al fracaso incluye el miedo a hacerlo mal (p. ej., la realización de una actividad o una interacción interpersonal), y el temor a las consecuencias de realizarlo mal (una evaluación negativa, una reprimenda, ser objeto de burla por parte de los demás, la no consecución de una meta, un despido, la vergüenza que origina, la disminución de la autoestima, la incertidumbre etc.), o simplemente el miedo a no saber qué va a pasar si se equivoca, es el miedo a lo desconocido, o miedo al futuro, señalado por distintos autores (Alonso \& Sánchez-Herrero, 2011; Gullone \& King, 1992; Gullone, 2000; Moreno-Murcia \& Conte, 2011).

Se han llevado a cabo distintos trabajos sobre el miedo en el ámbito laboral. En primer lugar, algunos autores analizan los miedos específicos que tienen algunos colectivos en el desempeño de su profesión, por ejemplo docentes (Collazos, González, \& González, 2012; González González, 2014). En segundo lugar, estarían los miedos de 
los trabajadores frente a diferentes situaciones que pueden presentarse en su ámbito laboral. Por ejemplo, situaciones críticas que afrontan en el trabajo, como personal sanitario de emergencias (Mikkola, Huhtala, \& Paavilainen, 2017) o expatriados (Giorgi,Montani, Fiz-Pérez, Arcangeli, \& Mucci, 2016). Por último, hay algunos trabajos que analizan los miedos que surgen cuando una persona se enfrenta a una nueva situación laboral, una transición, cuando acomete una nueva tarea, un nuevo puesto, etc. Los miedos pueden aparecer a lo largo de todo el desarrollo de carrera, pero pueden darse con más probabilidad al comienzo de la misma y en las diferentes transiciones que se dan en el ciclo vital y profesional del individuo. Estos estudios, aunque escasos, analizan el miedo de profesionales que tienen que afrontar por primera vez una tarea, por ejemplo, personal de enfermería recién graduado que siente ansiedad por el nuevo rol que tienen que asumir (Le, 2016), temores sobre si poseen las habilidades necesarias para la realización de prácticas (Haycock-Stuart, MacLaren, McLachlan, \& James, 2016), cuando tienen que enseñar habilidades clínicas a otros (Cangelosi, Crocker, \& Sorrell, 2009), al enfrentarse a la muerte de personas mayores (Terry \& Carroll, 2008), o cuando adoptan roles para los que no tienen experiencia (Gohery \& Meaney, 2013).

De forma similar, aparece el miedo en pasantes de abogacía (Klinker, Hoover, Valle, \& Hardin, 2014), en estudiantes de high school (Bernstein- Yamashiro, 2004; De Castella, Byrne, \& Covington, 2013), en estudiantes de psicología ante la práctica clínica con pacientes (Foladori, 2009) o en docentes (Collazos et al., 2012; González González, 2014). En bastantes de estos casos se menciona explícitamente la utilidad de la mentoría para ayudar a enfermeras, pasantes de abogacía, profesionales de éxito y estudiantes.

Existen diferentes instrumentos para medir el miedo de manera general. Probablemente las escalas más conocidas que permiten evaluar el miedo, junto con otras emociones, son las Escalas de Afecto Positivo y Negativo (PANAS), en las que el miedo es uno de los componentes del afecto negativo. Recientemente el PANAS se ha validado con una muestra española (López-Gómez, Hervás, \& Vázquez, 2015). Por otro lado, la escala Fear
Survey Schedule (FSS) de Gullone \& King (1992) recoge información sobre posibles fuentes de reacciones emocionales desadaptativas a la muerte, animales, situaciones interpersonales, ruidos, etc.

El Performance Failure Appraisal Inventory (PFAI) de Conroy, Willow , \& Metzler (2002) fue validado al contexto español por Moreno-Murcia y Conte (2011). La escala consta de 25 ítems, agrupados en cinco factores: miedo a experimentar vergüenza (p. ej., "Cuando me equivoco, me da vergüenza si los demás están allí para verlo"), miedo a la devaluación de uno mismo (p. ej., "Cuando no tengo éxito, me siento menos valioso que cuando tengo éxito"), miedo a tener un futuro incierto (p. ej., "Cuando me equivoco, creo que mis planes para el futuro cambiarán”), miedo a perder interés de los demás (p. ej., "Cuando no tengo éxito, algunas personas no se muestran interesadas por mí"), y el temor de perturbar a personas importantes (p. ej., "Cuando me equivoco, esto disgusta a la gente que me importa"). Las respuestas son cerradas y se responden a través de una escala tipo Likert, donde 1 corresponde a "no lo creo nada" y 5 "a lo creo al $100 \%$ ".

El grado en que una persona teme ser evaluado negativamente por los demás se ha medido a través de la Escala de Miedo a la Evaluación Negativa en su versión larga original (Watson \& Friend, 1969) y en su posterior versión breve (Leary, 1983), validada en España por Gallego et al. (2007).

De forma más específica, se ha evaluado el miedo en sujetos que se enfrentan a una nueva situación laboral. Así, Alonso y Sánchez-Herrero (2011) proponen y validan una escala para evaluar los miedos frente a la búsqueda y consecución de empleo, encontrando que es una variable multidimensional con distintas facetas: miedo a no dar la talla, miedo a quedar mal con otros, miedo a los procesos de selección, miedo a trabajar mucho, sin horarios y con normas rígidas, miedo a no poder desarrollar mi talento en el trabajo y miedo a desconocer el futuro.

No existen instrumentos para evaluar los miedos en profesionales que ejercen por primera vez un rol, aspecto especialmente relevante en aquellos casos que supone interacción social y en actividades de acompañamiento, por ejemplo, la que realiza un mentor. 
Los procesos de autorregulación del aprendizaje no siempre están adquiridos por parte de los estudiantes de nuevo ingreso cuando acceden a la universidad (Bruna, Pérez, Bustos, \& Núñez, 2017; García Ripa, Sánchez García, \& Risquez, 2015) y la mentoría o tutoría entre iguales puede jugar un papel importante a la hora de promover una actitud activa en estudiantes de nuevo ingreso y paliar los miedos de sus telémacos, ya que les ofrece una oportunidad para que descubran sus fortalezas y limitaciones sin tener miedo al fracaso, ni a ser juzgados, ni a represalias por expresar sus opiniones (Pomeroy \& Steiker, 2009), además de incrementar su confianza (López et al., 2013).

La literatura refleja cómo el mentor ayuda a superar los miedos de los telémacos, pero ¿y los miedos del mentor? ¿Qué temores tiene antes de afrontar el rol de mentor? En este caso las referencias son escasas. Hunt, McGee, Gutteridge y Hughes (2016) hacen alusión al miedo en los mentores, pero referido a comportamientos agresivos por parte de estudiantes.

El mentor, de la misma forma que la adopción de otros roles y retos profesionales por primera vez, es probable que muestre temor a enfrentarse a lo desconocido y al desconocido, lo que mina la relación de rapport inicial (Foladori, 2009).

En los programas de mentoría entre iguales que se llevan a cabo en universidades sería útil un instrumento que evalúe los miedos del mentor, que además de detectarlos sirviera de base para intervenir sobre los mismos. En la actualidad no hay una escala que permita evaluar los miedos del mentor.

Entendemos que los miedos del mentor se refieren a los diferentes temores a los que tiene que enfrentarse un estudiante en el desempeño de su rol como mentor en un programa de mentoría.

\section{Método}

\section{Objetivos}

El objetivo de esta investigación es la elaboración y posterior validación de una escala de miedos para utilizar en el entorno de la mentoría entre iguales en contextos universitarios.

\section{Población y Muestra}

La muestra tiene un carácter incidental y está formada por 748 estudiantes que ejercen el rol de mentores en la Universidad Complutense de Madrid (UCM). Se trata de distintas cohortes de estudiantes mentores de la UCM desde el curso 2012/13 al 2016/17.

Hay 467 mujeres (69.2\%) y 208 hombres (30.8\%), en 71 ocasiones no indicaron el sexo. Los estudiantes mentores están en segundo, tercero, o cuarto curso y su edad media es de 21.5 años $(S D=4.47)$, en 46 casos no indican su edad.

\section{Instrumento}

La revisión de la literatura nos proporciona una primera aproximación al concepto a medir. Sin embargo, dado que no hay nada específico al campo de la mentoría se analizaron áreas de contenido similares. Además, se examinaron tres instrumentos sobre miedos relacionados con la temática adaptados y/o creados en España, ya señalados anteriormente. Por último, se llevaron a cabo distintos estudios cualitativos (grupos de discusión) previos que recogieron diferentes miedos de los estudiantes en su rol de mentores. Esta información fue recogida tanto en los cursos de formación de mentores, que se llevan a cabo antes de la implantación del programa de mentoría, como en las sesiones de evaluación formativa (feedback) con los mentores por parte de los coordinadores del programa. Como consecuencia de lo anterior se obtuvieron 7 dimensiones del constructo con diferentes indicadores que se operativizaron en un conjunto inicial de 25 ítems que había que responder utilizando una escala Likert de 5 puntos (Tabla 1).

Con el fin de determinar la validez discriminante de la escala de miedos, se aplican tres instrumentos más:

La Escala de Engagement en Contexto Académico de Schaufeli y Bakker (2003). El engagement, lo definen Schaufeli, Salanova, González-Roma y Bakker (2002) como un estado mental positivo, de realización, relacionado con el trabajo que se caracteriza por vigor (energía y activación mental, voluntad para esforzarse y perseverar incluso ante dificultades), dedicación (involucración con la tarea, estar orgulloso e inspirado) y absorción (concentración, sentimiento de que el tiempo pasa rápidamente). Se pregunta a los sujetos por sus sentimientos en contexto universitario, pidiéndole el número de veces que se presenta cada afirmación (p. ej., 
Tabla 1. Matriz de especificaciones

\begin{tabular}{|c|c|c|}
\hline Fuente & Dimensiones & Indicadores \\
\hline \multirow[b]{2}{*}{$\begin{array}{l}\text { Revisión Literatura } \\
\text { y Instrumentos }\end{array}$} & Miedo a hacerlo mal & $\begin{array}{l}\text { A la realización de actividades } \\
\text { A las interacciones interpersonales } \\
\text { A no poder desarrollar mi talento } \\
\text { A perturbar a personas importantes }\end{array}$ \\
\hline & Miedo a no saber qué va a pasar & $\begin{array}{l}\text { A lo desconocido } \\
\text { Al futuro, al cambio } \\
\text { A lo que pasará si se equivoca } \\
\text { Reprimendas } \\
\text { Evaluación negativa } \\
\text { A ser objeto de burla o rechazo } \\
\text { A no conseguir una meta, a fracasar } \\
\text { A un despido } \\
\text { A la vergüenza que origina } \\
\text { A la disminución de autoestima } \\
\text { A trabajar mucho }\end{array}$ \\
\hline \multirow{4}{*}{$\begin{array}{l}\text { Recogida de Información } \\
\text { Cualitativa }\end{array}$} & Miedo no ser un buen mentor & $\begin{array}{l}\text { A la realización de reuniones de mentoría } \\
\text { A que no le guste la labor del mentor } \\
\text { A ofrecer información adecuada en las reuniones } \\
\text { A resolver las dudas que tengan }\end{array}$ \\
\hline & Miedo a lo que pasará en la mentoría & $\begin{array}{l}\text { A no reaccionar ante imprevistos } \\
\text { A lo inesperado } \\
\text { A no saber qué pasará }\end{array}$ \\
\hline & Miedo al coordinador del programa de mentoría & $\begin{array}{l}\text { A la evaluación negativa } \\
\text { A no consecución de créditos } \\
\text { A las críticas del coordinador }\end{array}$ \\
\hline & Miedo a no ganarse el respeto de los telémacos & $\begin{array}{l}\text { A interaccionar con otros } \\
\text { A perder el interés de los telémacos } \\
\text { A que abandonen }\end{array}$ \\
\hline
\end{tabular}

"Mis estudios me inspiran cosas nuevas").

La Escala de Autoeficacia General de Baessler y Schwarzer (1996) evalúa el sentimiento estable de competencia personal para manejar de forma eficaz una gran variedad de situaciones estresantes. La escala fue validada con población española por Sanjuán, Pérez y Bermúdez (2000); un ejemplo de ítem, al que hay que contestar señalando el grado de acuerdo en una escala Likert es: "Me es fácil persistir en lo que me he propuesto hasta llegar a alcanzar mis metas".

La Escala de Habilidades Sociales de Ferris, Witt y Hochwarter (2001), que pregunta por el grado de acuerdo sobre distintas afirmaciones (p. ej., "Sé que tengo que decir y hacer en las situaciones de interacción social") para evaluar las habilidades en las relaciones sociales.

\section{Procedimiento}

El programa de mentoría de la Universidad Complutense de Madrid tiene como objetivo facilitar la integración de los estudiantes de nuevo ingreso con la ayuda de estudiantes de cursos superiores que asumen el rol de mentor (www.ucm.es/mentorias).

Se trata de un programa formal en el que mentor y telémacos (estudiantes de primero que participan en el programa) tienen que realizar al menos cinco reuniones, con una frecuencia de unos 10 días. A cada mentor se le asignan entre 4 y 6 telémacos En la primera parte de la reunión se analizan cómo han ido las cosas desde que no se han visto y se abordan los temas más urgentes o necesarios para el telémaco en ese momento, solucionando las dudas que pueda tener. En la segunda parte de cada reunión se aborda un tema específico que se sabe será de interés para el telémaco: asignaturas y profesores, biblioteca, recursos informáticos, servicios de la universidad, becas, salidas laborales, etc. Los contenidos de las reuniones han sido elaborados, revisados y actualizados previamente por los alumnos mentores.

Para poder desempeñar adecuadamente su 
función reciben un curso de formación de ocho horas de duración con metodologías activas en las que priman la discusión de grupos y la realización de juegos de roles (los contenidos y la metodología del taller de formación puede consultarse en Alonso \& Calles, 2008). Por realizar el curso los mentores reciben un crédito y por la realización de las distintas reuniones 1,5 créditos.

Después de realizar el curso de formación y antes de la primera reunión con los telémacos, los mentores cumplimentan por e-mail la escala de miedos, otra de engagement, otra de habilidades sociales y otra de autoeficacia. La cumplimentación de dichas escalas es voluntaria, y se les informa que los datos personales recabados serán tratados de forma anónima, y que sólo van a ser utilizados con fines de investigación.

\section{Análisis estadísticos}

Los 748 casos de la muestra se partieron en dos submuestras de forma aleatoria, una de ellas se realizó el Análisis Factorial Exploratorio (AFE) y con la otra el Análisis Factorial Confirmatorio (AFC). Las distribuciones de las variables eran adecuadas ya que tenían índices de asimetría y curtosis inferiores 1,2, la escala tenía cinco categorías de respuesta y la muestra no es muy elevada, por lo que se siguieron las pautas de Lloret, Ferreres, Hernández y Tomás (2014) y se utilizó la matriz de correlaciones de Pearson para el AFE. El método de extracción de factores utilizado para el AFE fue máxima verosimilitud (ML) y promín de rotación oblicua, ya que permitía una solución factorial más simple y fácil de interpretar. Los análisis se realizaron con el programa FACTOR (Lorenzo-Seva \& Ferrando, 2006). El AFC fue realizado con el programa AMOS v.23.

Posteriormente, se analizó la invarianza factorial a través de AFC multigrupo para conocer si el instrumento mide el constructo de la misma forma en hombres y en mujeres, y en mentores que ya han realizado alguna reunión de mentoría frente a los que no lo han realizado. Por último, se realizaron análisis de correlaciones bivariadas entre las puntuaciones totales de miedo y los otros instrumentos que se aplicaron.

\section{Resultados}

Se realizaron distintas análisis factoriales exploratorios y se fueron tomando decisiones respecto a qué elementos permanecían en la escala definitiva. Se eliminaron ítems cuando el índice de homogeneidad corregido (correlación ítemtotal sin el ítem analizado) era inferior a .4; cuando en los sucesivos análisis factoriales realizados la comunalidad era inferior a .3 ; o cuando el peso del ítem en el factor era inferior a .3.

Finalmente, la solución más satisfactoria se encontró con 13 elementos que se agrupaban en 4 factores. El número de factores se decidió teniendo en cuenta la facilidad para interpretar la solución. Los nombres otorgados a cada factor han sido "miedo a equivocarme", "miedo a no saber qué va a pasar", "miedo a que no me respeten" y "miedo al superior". El alfa de Cronbach total de la escala con esos 13 elementos es de .90 , y el alfa de cada subescala está por encima de .73 .

La varianza total explicada con cuatro factores es del $70 \%$ de la información. El análisis paralelo recomienda un solo factor, lo que justifica el cálculo de una puntuación global. No obstante, la coherencia de los factores con lo reflejado en la literatura justifica que se haya optado por esta opción de mantener los 4 factores.

En el análisis final realizado la prueba KaiserMeyer-Olkin (KMO) ofrece un valor de .902, siendo la prueba de Bartlett significativa, $\chi^{2}$ $(78)=2350.8 ; p<.001$. Los datos indican, por tanto, la pertinencia del análisis factorial y el buen ajuste del modelo.

Se calcularon los distintos estadísticos descriptivos de los ítems finales, el alfa de Cronbach para la escala conjunta, las correlaciones ítem-total y los factores resultantes del AFE aparecen en la Tabla 2.

El AFC (ver Figura 1) pone de manifiesto el ajuste aceptable del modelo siguiendo los criterios propuestos por Herrero (2010), ya que la proporción entre $\chi^{2}$ y grados de libertad es cercano a tres, $\chi^{2}(59)=190.97, p<.001$; el comparative fit index (CFI) es de .95 (justo en el punto de corte para cumplir con la recomendación habitual de .95); el Tucker-Lewis index (TLI) tiene un valor de .93 (mayor de los .90 recomendados); el root 
Tabla 2. Análisis Factorial Exploratorio, medias, desviación estándar, coeficientes de asimetría y curtosis, comunalidades y correlaciones ítem total de la Escala de Miedos de Mentores

\begin{tabular}{|c|c|c|c|c|c|c|c|c|c|c|}
\hline & $\mathrm{F} 1$ & $\mathrm{~F} 2$ & F3 & $\mathrm{F} 4$ & M & SD & Asimetría & Curtosis & $\mathrm{h}^{2}$ & $\begin{array}{c}\mathrm{r} \\
\text { item } \\
\text {-total }\end{array}$ \\
\hline Factor 1. Miedo a equivocarme & & & & & 10.31 & 3.59 & & & & \\
\hline $\begin{array}{l}\text { 3. Tengo miedo a no saber llevar las } \\
\text { reuniones }\end{array}$ & .96 & & & & 2.57 & 1.13 & 0.23 & -0.89 & 0.81 & .70 \\
\hline $\begin{array}{l}\text { 18. Tengo miedo a no saber que decir } \\
\text { en las reuniones }\end{array}$ & .44 & .48 & & & 2.26 & 1.05 & 0.49 & -0.58 & 0.59 & .73 \\
\hline $\begin{array}{l}\text { 4. Tengo miedo a no saber responder a } \\
\text { todo lo que me pregunten }\end{array}$ & .43 & & & & 2.75 & 1.13 & 0.11 & -0.81 & 0.32 & .78 \\
\hline 8. Tengo miedo a hacerlo mal & .34 & & & & 2.73 & 1.24 & 0.18 & -1.06 & 0.47 & .76 \\
\hline $\begin{array}{l}\text { Factor } 2 \text {. Miedo a no saber qué va a } \\
\text { pasar }\end{array}$ & & & & & 6.64 & 2.64 & & & & \\
\hline $\begin{array}{l}\text { 19. Tengo miedo a no saber superar } \\
\text { situaciones imprevistas }\end{array}$ & & .95 & & & 2.3 & 1.06 & 0.47 & -0.59 & 0.80 & .75 \\
\hline $\begin{array}{l}\text { 20. Tengo miedo a no saber reaccionar } \\
\text { ante algún desafío }\end{array}$ & & .92 & & & 2.22 & 0.99 & 0.39 & -0.70 & 0.77 & .68 \\
\hline 21. Tengo miedo a la incertidumbre & & .45 & & & 2.11 & 0.97 & 0.57 & -0.34 & 0.43 & .88 \\
\hline Factor 3. Miedo a que no me respeten & & & & & 6.62 & 2.72 & & & & \\
\hline 24. Tengo miedo a que no me respeten & & & 1.06 & & 2.16 & 1.14 & 0.66 & -0.51 & 0.90 & .56 \\
\hline 23. Tengo miedo a parecer débil & & & .51 & & 1.98 & 1.07 & 0.93 & 0.11 & 0.56 & .68 \\
\hline $\begin{array}{l}\text { 17. Tengo miedo a que no valoren } \mathrm{mi} \\
\text { trabajo o mi persona }\end{array}$ & & & .35 & .35 & 2.48 & 1.09 & 0.21 & -0.77 & 0.41 & .79 \\
\hline Factor 4. Miedo al superior & & & & & 5.62 & 2.31 & & & & \\
\hline $\begin{array}{l}\text { 13. Tengo miedo a tener un profesor- } \\
\text { tutor crítico }\end{array}$ & & & & .97 & 1.86 & 0.98 & 1.04 & 0.47 & 0.74 & .54 \\
\hline $\begin{array}{l}\text { 16. Tengo miedo a no saber reaccionar } \\
\text { ante el profesor-tutor }\end{array}$ & & & & .61 & 1.89 & 0.95 & 0.83 & -0.17 & 0.50 & .65 \\
\hline $\begin{array}{l}\text { 12. Tengo miedo a la responsabilidad } \\
\text { que supone ser mentor }\end{array}$ & & & & .38 & 1.87 & 0.93 & 0.95 & 0.54 & 0.43 & .74 \\
\hline Alfa de Cronbach total $=.905$ & .80 & .84 & .76 & .74 & 29.19 & 9.40 & & & & \\
\hline
\end{tabular}

Tabla 3. Modelos de invarianza factorial en función del sexo en la muestra total

\begin{tabular}{|c|c|c|c|c|c|c|c|}
\hline & $\partial^{2}(\mathrm{gl})$ & $\Delta X^{2}$ & CFI & $\Delta \mathrm{CFI}$ & TLI & RMSEA & AIC \\
\hline 1.Configural & $393.62(118)$ & -- & .93 & -- & .91 & .06 & 521.62 \\
\hline 2.Métrica & $405.43(127)$ & 11.81 & .93 & 0 & .92 & .06 & 515.43 \\
\hline 3.Escalar & 419.83 (137) & 14.40 & .93 & 0 & .92 & .06 & 509.83 \\
\hline 4.Estricta & $446.98(150)$ & 27.15 & .93 & 0 & .93 & .05 & 510.98 \\
\hline
\end{tabular}

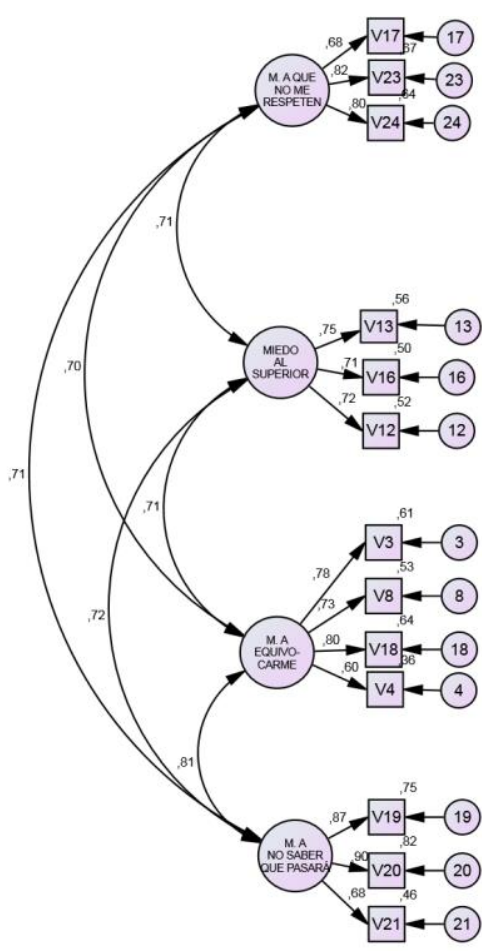

Figura 1. Análisis Factorial Confirmatorio de la Escala de Miedos del Mentor mean square error of approximation (RMSEA) tiene un valor de .077 (lo ideal es por debajo de .05 ; menor de .08 indica que $90 \%$ IC<.10), y el parsimonious normed fit index (PNFI) igual a .7 (que está en el intervalo recomendado). Con trece ítems se confirman los cuatro factores encontrados en el AFE.

Después, se realizó un AFC multigrupo para analizar la invarianza factorial en función del sexo. La diferencia ente los valores de la Tabla 2, en el índice comparativo de Bentler (CFI) entre cada dos modelos anidados es inferior a 0.01, por lo que se aceptan todos los modelos. Además, las diferencias en $\chi^{2}$ y en AIC no muestran grandes variaciones entre un modelo y el siguiente, CFI y TLI tienen valores superiores a .90 y el RMSEA es inferior a .08. Por todo ello, se puede concluir que existe invarianza configural, métrica, escalar y estricta entre los hombres y mujeres de la muestra (Tabla 3). 
Tabla 4. Modelos de invarianza factorial en función de si han realizado alguna reunión de mentoría

\begin{tabular}{lccccccc}
\hline & $\chi^{2}(\mathrm{gl})$ & $\Delta \chi^{2}$ & CFI & $\Delta$ CFI & TLI & RMSEA & AIC \\
\hline 1.Configural & 409.09 & --- & .93 & --- & .91 & .06 & 537.09 \\
2.Métrica & 415.96 & 6.87 & .93 & 0 & .92 & .06 & 525.96 \\
3.Escalar & 422.95 & 6.99 & .93 & 0 & .92 & .06 & 512.95 \\
4.Estricta & 440.2 & 17.25 & .93 & 0 & .93 & .06 & 504.20 \\
\hline
\end{tabular}

Tabla 5. Miedos del mentor segmentadas en función del género y del número de reuniones realizadas

\begin{tabular}{|c|c|c|c|c|c|c|c|c|c|c|c|c|}
\hline \multirow[b]{2}{*}{ Género } & \multirow[b]{2}{*}{ Reuniones Realizadas } & \multirow[b]{2}{*}{$\mathrm{N}$} & \multicolumn{2}{|c|}{$\begin{array}{c}\text { Miedo a } \\
\text { equivocarme }\end{array}$} & \multicolumn{2}{|c|}{$\begin{array}{c}\text { Miedo a no } \\
\text { saber qué va a } \\
\text { pasar }\end{array}$} & \multicolumn{2}{|c|}{$\begin{array}{l}\text { Miedo a que no } \\
\text { me respeten }\end{array}$} & \multicolumn{2}{|c|}{$\begin{array}{c}\text { Miedo al } \\
\text { superior }\end{array}$} & \multicolumn{2}{|c|}{ Miedo Total } \\
\hline & & & $\mathrm{M}$ & SD & $\mathrm{M}$ & SD & $\mathrm{M}$ & $\mathrm{SD}$ & $\mathrm{M}$ & SD & $\mathrm{M}$ & $\mathrm{SD}$ \\
\hline \multirow{4}{*}{ Hombre } & Ninguna & 166 & 2.34 & .80 & 2.03 & .82 & 1.97 & .81 & 1.64 & .67 & 2.02 & .62 \\
\hline & 1 o 2 reuniones & 29 & 1.90 & .77 & 1.68 & .70 & 1.84 & .83 & 1.39 & .56 & 1.72 & .61 \\
\hline & Total & 195 & 2.27 & .81 & 1.98 & .81 & 1.95 & .81 & 1.60 & .66 & 1.97 & .63 \\
\hline & Ninguna & 369 & 2.70 & .94 & 2.32 & .91 & 2.39 & .96 & 1.93 & .82 & 2.36 & .78 \\
\hline \multirow[t]{3}{*}{ Mujer } & 1 o 2 reuniones & 65 & 2.63 & .95 & 2.25 & .92 & 2.22 & .91 & 1.87 & .78 & 2.25 & .72 \\
\hline & Total & 434 & 2.69 & .94 & 2.31 & .91 & 2.37 & .96 & 1.92 & .81 & 2.34 & .77 \\
\hline & Ninguna & 535 & 2.59 & .91 & 2.23 & .89 & 2.26 & .94 & 1.84 & .79 & 2.25 & .75 \\
\hline \multirow[t]{2}{*}{ Total } & 1 o 2 reuniones & 94 & 2.41 & .96 & 2.07 & .89 & 2.10 & .90 & 1.72 & .75 & 2.08 & .73 \\
\hline & Total & 629 & 2.56 & .92 & 2.21 & .89 & 2.24 & .93 & 1.82 & .78 & 2.23 & .75 \\
\hline
\end{tabular}

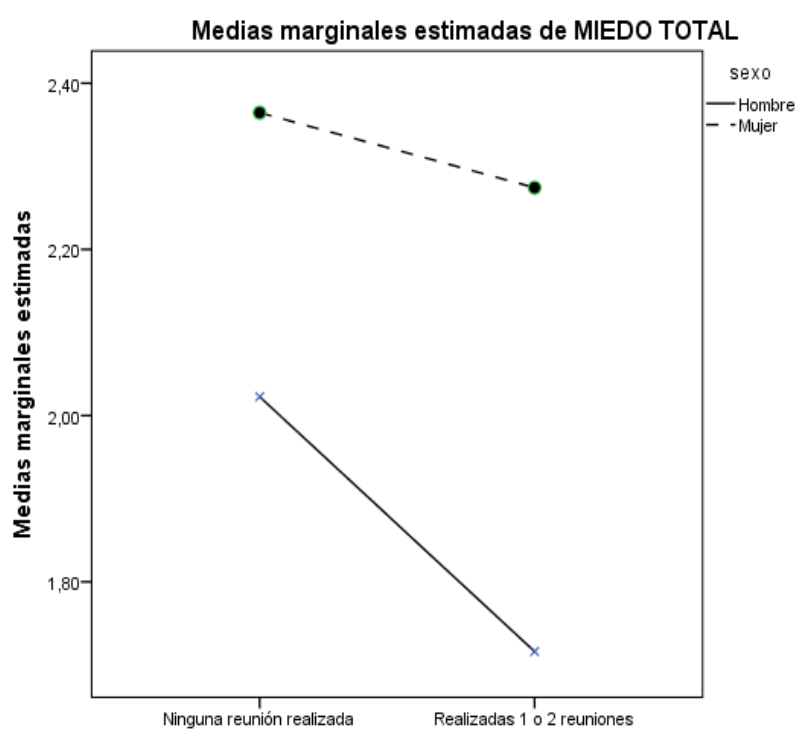

Ha tenido reuniones

Figura 2. Puntuación total del miedo en función del sexo y si había realizado reuniones previamente

El AFC multigrupo para analizar la invarianza factorial en función de si habían realizado o no alguna reunión de mentoría (Tabla 4) también pone de manifiesto el alto grado de acuerdo en las estructuras factoriales con equivalencia factorial estricta.

El ANOVA realizado en función del género y de si habían realizado alguna reunión con sus telémacos, tomando como variable criterio la puntuación total de miedos (calculada con la media de las 13 variables del modelo) pone de manifiesto que existen diferencias estadísticamente significativas para la variable del género, $F(1$, $625)=26.54, p<.001, \eta^{2}=.04$; puntuando las mujeres más alto que los hombres (ver Tabla 5 y Figura 2), lo que indica que tienen más miedo que ellos. También se encuentran diferencias entre los que habían realizado reuniones frente a los que no lo habían hecho, $F(1,625)=5.16, p<.05, \eta^{2}=.008$; los que todavía no habían afrontado la situación tenían mayor miedo. No hay diferencias en la interacción género y realización de reuniones, $F$ $(1,625)=1.53, p=.22, \eta^{2}=.002$.

Esa misma tendencia se produce con las distintas dimensiones o factores de miedo, hay diferencias estadísticamente significativas entre hombres y mujeres en "miedo a equivocarme", $F(1,625)=25.88, p<.001, \eta^{2}=.04$; "miedo a no saber qué va a pasar", $F(1,625)=16.4, p<.001$, $\eta^{2}=.03$; "miedo a que no me respeten", $F(1,625)=$ 
Tabla 6. Correlaciones de Pearson entre las dimensiones de miedo. autoeficacia. habilidades sociales y engagement

\begin{tabular}{lccccc}
\hline & $\begin{array}{c}\text { Miedo a } \\
\text { equivocarse }\end{array}$ & $\begin{array}{c}\text { Miedo a no saber } \\
\text { qué va a pasar }\end{array}$ & $\begin{array}{c}\text { Miedo a que no } \\
\text { me respeten }\end{array}$ & $\begin{array}{c}\text { Miedo al } \\
\text { superior }\end{array}$ & Miedo Total \\
\hline Autoeficacia & $-.338^{* *}$ & $-.357^{* *}$ & -.202 & -.219 & $-.330^{* *}$ \\
Habilidades & $-.430^{* *}$ & $-.399^{* *}$ & $-.377^{* *}$ & $-.385^{* *}$ & $-.461^{* *}$ \\
Sociales & $-.286^{*}$ & $-.345^{* *}$ & $-.305^{*}$ & -.229 & $-.337^{*}$ \\
Vigor & -.036 & -.121 & -.08 & -.146 & -.103 \\
Dedicacion & -.115 & -.105 & -.126 & -.024 & -.109 \\
Absorcion & \multicolumn{5}{c}{}
\end{tabular}

$13.15, p<.001, \eta^{2}=.02 ; \mathrm{y}$ “miedo al superior”, $F(1$, $625)=16.83, p<.001, \eta^{2}=.03$; en todos los casos las puntuaciones de las mujeres son mayores.

También hay diferencias significativas entre aquellos que ya han realizado al menos una reunión y aquellos que no lo han hecho, los segundos tienen más miedo que los primeros en "miedo a equivocarme", $F(1,625)=5.51, p<.05$, $\eta^{2}=.01$ y "miedo a no saber qué va a pasar", $F(1$, $625)=3.98, p<.05, \eta^{2}=.01$. No hay diferencias en los factores "miedo a que no me respeten", $F(1$, $625)=1.95, p=.16, \eta^{2}=.003$ y "miedo al superior", $\mathrm{F}(1, \quad 625)=2.70, \quad p=.10, \quad \eta^{2}=.004$. No hay diferencias en la interacción en las cuatro dimensiones de miedos.

Por último, las correlaciones con las otras tres variables medidas ponen de manifiesto que la puntuación total de miedo se relaciona negativamente con las tres pero con diferencias según los factores de miedo. A menor autoeficacia mayor miedo a equivocarse y mayor miedo a no saber qué va a pasar; sin embargo, no parece haber relación entre autoeficacia y los otros dos tipos de miedo. Cuanto menores son las habilidades sociales mayor es el miedo, y este efecto se produce en las cinco puntuaciones de miedo calculadas. $\mathrm{Y}$ respecto a las tres dimensiones de engagement, aparece una relación negativa y significativa con vigor en cuatro de las cinco puntuaciones de miedos (no se produce en el miedo al superior). No parece haber relación entre los miedos y las dimensiones de dedicación y absorción (ver Tabla 6).

\section{Discusión}

El objetivo del presente estudio es crear y validar un cuestionario que permita evaluar los miedos a los que se enfrentan los mentores en los programas de mentoría entre iguales en la Universidad.
Los datos obtenidos ponen de manifiesto una estructura con cuatro factores o facetas de miedo encontradas que se relacionan con las mencionadas en la revisión teórica. Así, el "miedo a equivocarme" refleja el miedo al fracaso o a fallar (Cacciotti, \& Hayton, 2015; Conroy, 2003; Gullone \& King, 1992; Jericó, 2006; Scherer \& Nakamura, 1968; Torregrosa, Murcia, \& Moreno, 2015), el "miedo a no saber qué va a pasar" se relaciona con el denominado miedo al futuro (Alonso \& Sánchez-Herrero, 2000; Gullone \& King, 1992; Gullone, 2000; Moreno-Murcia \& Conte, 2011), el "miedo a que no me respeten" con el miedo a las interacciones sociales o el miedo al rechazo (Gordillo et al., 2015; Jericó, 2006, de la Rubia et al., 2013) y el "miedo al superior" (Bedoya \& García, 2016).

El análisis de invarianza realizado ha permitido comprobar que los cuatro factores se ajustan a hombres y mujeres y a tener experiencia o no en la realización de reuniones de mentoría. Además, los factores se relacionan entre sí y tiene sentido calcular también una única puntuación total de miedos.

Los datos encontrados muestran que las mujeres tienen mayores miedos que los hombres a asumir al rol de mentor, lo que coincide con otras investigaciones en las que las mujeres expresan miedo con mayor frecuencia (p. ej., Pulido \& Herrera, 2016; Valdez et al., 2010) e intensidad (p. ej., Pérez \& Reidl, 2013) que los hombres. De forma similar, cuando el mentor ya ha realizado alguna reunión de mentoría, su miedo es menor que cuando todavía no se ha enfrentado a dicha situación (situaciones parecidas se han encontrado entre los miedos que afrontan las personas que buscan trabajo por primera vez y aquellas que ya lo han hecho previamente (Castaño, Alonso, \& Sánchez-Herrero, 2016).

Respecto a las relaciones de los miedos con 
otras variables, cuando la autoeficacia es más elevada el miedo a equivocarse y el miedo a no saber qué va a pasar son menores. También hay una relación inversa entre habilidades sociales y miedos (aspecto encontrado en otras investigaciones, por ejemplo la de Pulido \& Herrera, 2016) y respecto a la dimensión de "vigor" de la escala de engagement en contexto académico. El miedo influye negativamente en la voluntad de esforzarse y perseverar, pero no en la dedicación y la absorción, quizás porque estos dos están más relacionados con los sentimientos y la primera con intención de esfuerzo, lo que en caso de miedo intenso podría llevar a no asumir la responsabilidad de ejercer de mentor.

En consecuencia, esta investigación aporta un instrumento válido que permite medir los miedos de mentores antes de afrontar la primera reunión de mentoría con sus telémacos.

Detectar y trabajar los temores de los mentores nóveles puede ser un aspecto importante en los programas de mentoría. Dicha intervención puede integrarse de forma natural en un taller de formación genérico, incluyendo un espacio para facilitar la verbalización de los temores ante los otros miembros del grupo, así como la realización de juegos de rol que permitan vivir la experiencia de afrontar las situaciones que más miedo dan. El abordaje de los miedos en la formación de profesionales que asumen el rol de orientar a otros parece necesario, y no suele estar incluido en los programas de formación, sirva como ejemplo la ausencia en la formación de docentes. Abordar los temores de los docentes, por seguir con el ejemplo, permitiría conocer sus preocupaciones y así saber cómo ayudarles (Conway \& Clark, 2003).

Por otro lado, parece que sería útil en la formación de mentores, además de dedicar tiempo a reducir los miedos, hacerlo en elevar la autoeficacia, las habilidades sociales y los niveles de energía y activación mental de cara a afrontar el rol de mentor.

Parece que los mentores teme cuatro cosas: equivocarse, no controlar lo que pasará, que le rechacen, y a su superior; ser consciente de sus miedos es el primer paso para trabajarlos y tener éxito en el ejercicio de su rol.

Como limitaciones del estudio se puede señalar que la muestra se centra en un programa de mentoría de una única universidad. Sería conveniente analizar otras muestras de diferentes universidades. Los datos han sido recogidos de forma on-line a través de una página web, con las ventajas e inconvenientes que eso supone. Por otra parte, apenas hay investigación previa sobre miedos en mentores, lo que ha provocado que haya que sustentar la revisión teórica en miedos de otros tipos de profesionales. Otro aspecto interesante sería el efecto que el miedo de los mentores tiene sobre los telémacos, por ejemplo, sobre su satisfacción o su abandono del programa.

En futuras investigaciones se puede validar el instrumento con otros profesionales que acompañan y orientan como profesores, tutores, coaches, o mentores en organizaciones distintas a la universidad; creemos que la escala con pequeñas adaptaciones sería válida para dichos colectivos.

\section{Referencias}

Alonso, M. A., \& Calles, A. M. (2008). La formación de alumnos mentores. Mentoring \& Coaching, 1, 13-26. Retrieved from http://innovacioneducativa.upm.es/web_revist a/Revista_08.pdf

Alonso, M. A., \& Sánchez-Herrero, S. A. (2011). Elaboración y validación de una escala para diagnosticar los miedos en el proceso de búsqueda de empleo. Revista Española de Orientación y Psicopedagogía, 22(1), 80-92. doi:10.5944/reop.vol.22.num.1.2011.79

Baessler, J., \& Schwarzer, R. (1996). Evaluación de la autoeficacia: Adaptación española de la Escala de Autoeficacia General. Ansiedad y Estrés, 2, 1-8.

Bedoya, C., \& García, M. (2016). Efectos del miedo en los trabajadores y la organización. Estudios Gerenciales, 32, 60-70 doi:10.1016/j.estger.2015.10.002

Bernstein- Yamashiro, B. (2004). Learning relationships: Teacher- student connections, learning, and identity in high school. New Directions for Youth Development, 2004(103), 55-70. doi:10.1002/yd.91

Bruna, D., Pérez, M. V., Bustos, C., \& Núñez, J. C. (2017). Propiedades psicométricas del Inventario de Procesos de Autorregulación del 
Aprendizaje en estudiantes universitarios Chilenos. Revista Iberoamericana de Diagnóstico y Evaluación - e Avaliação Psicológica, 2(44), 77-91.

Cacciotti, G., \& Hayton, J. C. (2015). Fear and entrepreneurship: A review and research agenda. International Journal of Management Reviews, 17(2), 165-190.

doi:10.1111/ijmr.12052

Cangelosi, P., Crocker, S., \& Sorrell, J. M. (2009). EXPERT to NOVICE: Clinicians learning new roles as clinical nurse educators. Nursing Education Perspectives, 30(6), 367-71. Retrieved from http://0search.proquest.com.cisne.sim.ucm.es/docvie w/236585148? accountid $=14514$

Castaño, G., Alonso, M. A., \& Sánchez-Herrero (2016). Overcoming fears by establishing mini-tasks and activities as a strategy for enhancing career development prospects. In T. V. Martin (Ed.). Career Development. Theories, Practices and Challenges. New York: Nova Sciences Publishers.

Collazos Osorio, C., González Rouillé, L., \& González González, M. (2012). Los lenguajes del poder. Miedos de los Docentes. Plumilla Educativa, O(10), pp. 238-253. Retrieved from http://revistasum.umanizales.edu.co/ojs/index. php/plumillaeducativa/article/view/468/563

Conroy, D. E. (2001). Fear of failure: An exemplar for social development research in sport. Quest, 53(2), 165-183. doi:10.1080/00336297.2001.10491736

Conroy, D. E. (2003). Representational models associated with fear of failure in adolescents and young adults. Journal of Personality, 71(5), 757-784.

doi:10.1111/1467-6494.7105003

Conroy, D. E., Willow, J. P., \& Metzler, J. N. (2002). Multidimensional fear of failure measurement: The performance failure appraisal inventory. Journal of Applied Sport Psychology, 14(2), 76-90. doi:10.1080/10413200252907752

Conway, P. F., \& Clark, C. M. (2003). The journey inward and outward: A reexamination of Fuller's concerns-based model of teacher development. Teaching and Teacher Education, 19, 465-482. doi:10.1016/S0742-051X(03)00046-5
De Castella, K., Byrne, D., \& Covington, M. (2013). Unmotivated or motivated to fail? A cross-cultural study of achievement motivation, fear of failure, and student disengagement. Journal of Educational Psychology, 105(3), 861-880. doi:10.1037/a0032464

De la Rubia, 1. R., Cadena, C. H. G., \& Casas, C. (2013). Validación de la escala de ansiedad en la interacción social en estudiantes universitarios. Pensamiento Psicológico, 11(1), 27-42. Retrieved from http://0search.proquest.com.cisne.sim.ucm.es/docvie w/1418691434? accountid $=14514$

Ferris, G. R., Witt, L. A., \& Hochwarter, W. A. (2001). Interaction of social skill and general mental ability on job performance and salary. Journal of Applied Psychology, 86(6), 1075-1082. doi:10.1037/0021-9010.86.6.1075

Foladori, H. (2009). Initial fears in psychology students at the beginning of their professional practice in clinical psychology. Terapia psicológica, 27(2), 161-168.

doi:10.4067/S0718-48082009000200002

Gallego, M. J., Botella, C., García-Palacios, A., Quero, S., \& Baños, R. M. (2010). La escala de autoverbalizaciones durante la situación de hablar en público (SSPS): Propiedades psicométricas en una muestra española de fóbicos sociales. Psicología Conductual, 18(2), 343-363. Retrieved from http://0search.proquest.com.cisne.sim.ucm.es/docvie w/927580372? accountid $=14514$

Garcia-Ripa, M. I., Sanchez-Garcia, M. F., \& Risquez, A. (2016). Estrategias de aprendizaje y autorregulación motivacional. Identificación de perfiles para la orientación de estudiantes universitarios de nuevo ingreso. Revista Iberoamericana de Diagnóstico y Evaluación - e Avaliação Psicológica, 41(1), 39-57.

Giorgi, G., Montani, F., Fiz-Perez, J., Arcangeli, G., \& Mucci, N. (2016). Expatriates' multiple fears, from terrorism to working conditions: Development of a model. Frontiers in Psychology, 7. doi:10.3389/fpsyg.2016.01571

Gohery, P., \& Meaney, T. (2013). Nurses' role transition from the clinical ward environment to the critical care environment. Intensive \& Critical Care Nursing, 29(6), 321-328. doi:10.1016/j.iccn.2013.06.002 
González-González, M. A. (2014). Metáforas y paradojas de los miedos en los sujetos docentes. Revista Latinoamericana de Ciencias Sociales, Niñez y Juventud, 12(1), 355-370. doi:10.11600/1692715x.12121052013

Goldsmith, B. (2008). Dealing with fear in the workplace. Cost Engineering, 50(12), 21-22. Retrieved from http://0search.proquest.com.cisne.sim.ucm.es/docvie $\mathrm{w} / 220461092$ ?accountid $=14514$

Gordillo, F., Mestas, L., Arana, J.M., \& Salvador, J. (2015). El miedo como constructo de análisis sistemático. Alternativas en Psicología, 32, 27-47. Retrieved from http://bit.ly/2ENFueJ

Gullone, E. (2000). The development of normal fear: A century of research. Clinical Psychology Review, 20(4), 429-451. doi:10.1016/S0272-7358(99)00034-3

Gullone, E., \& King, N. J. (1992). Psychometric evaluation of a revised fear survey schedule for children and adolescents. Journal of Child Psychology and Psychiatry, and Allied Disciplines, 33(6), 987-998. doi:10.1111/j.1469-7610.1992.tb00920.x

Haycock-Stuart, E., MacLaren, J., McLachlan, A., \& James, C. (2016). These terrifying three words: A qualitative, mixed methods study of students' and mentors' understandings of 'fitness to practise'. Nurse Education Today, 43, 15-22.

doi:10.1016/j.nedt.2016.04.017

Herrero, J. (2010). El análisis factorial confirmatorio en el estudio de la estructura y estabilidad de los instrumentos de evaluación: Un ejemplo con el cuestionario de autoestima CA-14. Psychosocial Intervention, 19, 289300. Recuperado en 12 de febrero de 2018, de http://scielo.isciii.es/scielo.php?script=sci_artt ext\&pid=S113205592010000300009\&lng=es\&tlng=es.

Hunt, L., McGee, P., Gutteridge, R., \& Hughes, M. (2016). Manipulating mentors' assessment decisions: Do underperforming student nurses use coercive strategies to influence mentors' practical assessment decisions? Nurse Education in Practice, 20, 154-162. doi:10.1016/j.nepr.2016.08.007

Jericó, P. (2006). No Miedo: En la empresa y en la vida. Barcelona: Alienta Editorial
Kish-Gepharta, J. J., Detert, J. R., Treviño, L. K., \& Edmondson, A. C. (2009). Silenced by fear: The nature, sources, and consequences of fear at work. Research in Organizational Behavior, 29, 63-193. doi:10.1016/j.riob.2009.07.002

Klinker, J. F., Hoover, J. D., Valle, F., \& Hardin, F. (2014). Decision making for democratic leadership in a guided internship. Planning and Changing, 45(1), 187-209. Retrieved from http://0search.proquest.com.cisne.sim.ucm.es/docvie w/1719260953 ?accountid $=14514$

Le, D. (2016). Meeting the needs of new graduate nurse practitioners. Journal of Doctoral Nursing Practice, 9(1), 88. doi:10.1891/2380-9418.9.1.88

Leary, M. R. (1983). Understanding social anxiety: Social, personality, and clinical perspectives. Beverly Hills [etc.]: Sage.

Lloret, S., Ferreres, A., Hernández, A., \& Tomás, I. (2014). El análisis factorial exploratorio de los ítems: Una guía práctica, revisada y actualizada. Anales de Psicología, 30, 11511169. doi:10.6018/analesps.30.3.199361

López, M., Santos, I., Bravo, A., \& Valle, J. F. del. (2013). El proceso de transición a la vida adulta de jóvenes acogidos en el sistema de protección infantil. Anales de Psicología, 29(1), 187-196.

https://dx.doi.org/10.6018/analesps.29.1.130542

López-Gómez, I., Hervás, G., \& Vázquez, C. (2015). Adaptación de las "escalas de afecto positivo y negativo" (PANAS) en una muestra general española. Psicología Conductual, 23(3), 529-548. Retrieved from http://0search.proquest.com.cisne.sim.ucm.es/docvie w/1749659761 ?accountid $=14514$

Lorenzo-Seva, U., \& Ferrando, P. J. (2006). ACTOR: A computer program to fit the exploratory factor analysis model. Behavior Research Methods, 38, 88-91. doi:10.3758/BF03192753

Mikkola, R., Huhtala, H., \& Paavilainen, E. (2017). Work- related fear and the threats of fear among emergency department nursing staff and physicians in Finland. Journal of Clinical Nursing, 26(19-20), 2953-2963. doi:10.1111/jocn.13633 
Molina, J., Chorot, P., Valiente, R. M., \& Sandín, B. (2014). Miedo a la evaluación negativa, autoestima y presión psicológica: Efectos sobre el rendimiento deportivo en adolescentes. Cuadernos De Psicología Del Deporte, 14(3), 57-66. Retrieved from http://0-

search.proquest.com.cisne.sim.ucm.es/docvie w/1658874402? accountid $=14514$

Moreno-Murcia, J. A., \& Conte, L. (2011). Predicción del miedo a equivocarse en jugadores de baloncesto a través del clima tarea de los iguales y la motivación intrínseca. Revista Mexicana de Psicología, 28(1), 43-52. Retrieved from http://www.redalyc.org/articulo.oa?id=243029 630004> ISSN 0185-6073

Orejudo, S., Fernández-Turrado, T., \& Briz, E. (2012). Resultados de un programa para reducir el miedo y aumentar la autoeficacia para hablar en público en estudiantes universitarios de primer año. Estudios Sobre Educación, 22, 199-217. Retrieved from http://0-

search.proquest.com.cisne.sim.ucm.es/docvie w/1467534666? accountid=14514

Perez, V. R., \& Reidl, L. M. (2013). Miedo y afrontamiento en adolescentes mexicanos. Psicogente, 16(30), 280-295. Retrieved from http://oaji.net/articles/2017/17871485547485.pdf

Pomeroy, E. C., \& Steiker, L. H. (2011). Paying it forward: On mentors and mentoring. Social Work, 56(3), 197-199. doi:10.1093/sw/56.3.197

Pulido, F., \& Herrera, F. (2016). Predictores del miedo y las habilidades sociales en adolescentes del contexto pluricultural de Ceuta. Acción Psicológica, 13(1), 179-190. doi:10.5944/ap.13.1.15560

Sanjuán Suárez, P., Pérez García, A. M., \& Bermúdez Moreno, J. (2000). Escala de autoeficacia general: Datos psicométricos de la adaptación para población española. Psicothema, 12(2), 509-513.

Schaufeli, W., \& Bakker, A. (2003). Utrecht Work Engagement Scale. Holanda: Utrecht University.

Schaufeli, W., Salanova, M., González-Romá, V., \& Bakker, A. (2002). The measurement of engagement and burnout: A two sample confirmatory factor analytic approach. Journal of Happiness Studies, 3(1), 71-92. doi:10.1023/a:1015630930326

Scherer, M. W., \& Nakamura, C. Y. (1968). A fear survey schedule for children (FSS-FC): A factor analytic comparison with manifest anxiety (CMAS). Behaviour Research and Therapy, 6(2), 173-182. doi:10.1016/0005-7967(68)90004-1

Terry, L. M., \& Carroll, J. (2008). Dealing with death: First encounters for first-year nursing students. British Journal of Nursing, 17(12), 760-765. doi:10.12968/bjon.2008.17.12.30298

Torregrosa, Y. S., Murcia, J. A. M., \& Moreno Murcia, J. A. (2015). Miedo a equivocarse y motivación autodeterminada en estudiantes adolescentes. Daimon, 15(3), 65-74. doi:10.4321/S1578-84232015000300006

Valdez, J. L., López, I., Torres, O., Piña, M., González, N. I., \& Maya, M. U. (2010). Los tipos de miedo prevalentes por generación y por sexo. Revista Electrónica de Psicología Iztacala, 13(4), 163-181. Retrieved from http://www.iztacala.unam.mx/carreras/psicolo gia/psiclin/vol13num4/Vol13No4Art10.pdf

Watson, D., \& Friend, R. (1969). Measurement of social-evaluative anxiety. Journal of Consulting and Clinical Psychology, 33(4), 448-457. doi:10.1037/h0027806 